\title{
OXIDAÇÃO LIPÍDICA EM PEIXES: MECANISMO DE AÇÃO E PREVENÇÃO
}

\section{Lipid oxidation in fishes: action mechanism and prevention}

FOGAÇA, F.H.S. ${ }^{1}$; SANT'ANA, L.S. ${ }^{2}$

\begin{abstract}
1 Empresa Brasileira de Pesquisa Agropecuária, Centro de Pesquisa Agropecuária do Meio-Norte (CPAMN)
2 Centro de Aqüicultura da UNESP (CAUNESP)
\end{abstract}

\section{RESUMO}

A aquicultura é uma atividade destinada a produzir alimentos de alto valor nutritivo, porém suas condições ambientais e seu manejo aumentam as reações de estresse nos peixes, prejudicando a qualidade de seus produtos. A oxidação lipídica é uma das principais causas de deterioração do pescado, devido ao alto grau de insaturação dos ácidos graxos dos organismos aquáticos. Esta revisão descreve os principais mecanismos desencadeadores da oxidação lipídica, seus métodos de análise e algumas medidas preventivas, dando enfase à utilização in vivo da vitamina $\mathrm{E}$ como substância antioxidante em peixes.

Palavras-chave: ácidos graxos insaturados; antioxidantes; propagação; vitamina E

\section{ABSTRACT}

Aquaculture produces food with high nutritive value, however its environmental conditions and management increase the stress reaction of fishes, prejudicing the quality of their products. The lipid oxidation is one of the main causes of the fishery deterioration, due the high unsaturated value of fatty acid of aquatic organisms. This review describes lipid oxidation mechanisms, their analysis methodology and some preventive ways, emphasizing the antioxidants effects of vitamin E utilization in fish's diet.

Key words: antioxidant; propagation; vitamin E; unsaturated fatty acids 


\section{INTRODUÇÃO}

Há pelo menos uma década, as taxas de crescimento da aquicultura mantêm-se em patamares superiores a $10 \%$ ao ano (FAO, 2003a), elevando a sua atual importância na oferta mundial de pescados (Borghetti, 2003). Segundo a Food and Agricultural Organization, a produção mundial de pescados em 2006 foi estimada em 132,52 milhões de toneladas, sendo que desse total, 30,77 milhões de toneladas foram provenientes do cultivo de espécies de água doce. A produção brasileira contribuiu com 388.747 toneladas produzidas pela aquicultura, totalizando aproximadamente 1.086.504 toneladas de peixes em 2006 (FAOSTAT, 2006).

A aquicultura é uma atividade destinada a produzir alimentos de alto valor nutritivo, porém suas condições ambientais e seu manejo aumentam as reações de estresse nos peixes, prejudicando a qualidade de seus produtos (Macedo-Viegas e Souza, 2004). A oxidação lipídica é o processo primário de deterioração da qualidade dos peixes, manifestando-se por mudanças em seu cheiro, cor, textura, valor nutritivo, e possível produção de compostos tóxicos (Jensen et al., 1998), afetando sua aceitabilidade para o consumo (NogalaKalucka et al., 2005). É acentuada imediatamente após o abate e durante o processamento, quando ocorre a destruição da integridade das membranas celulares pelo corte das carnes, facilitando a propagação das reações oxidativas (Morrissey et al., 1998).

\section{A Deterioração do Pescado e a Oxidação Lipídica}

O pescado é extremamente perecível devido às características intrínsecas de sua carne, como: elevada atividade de água, composição química, teores de gorduras insaturadas facilmente oxidáveis e, principalmente ao $\mathrm{pH}$ próximo da normalidade (Melo Franco e Landgraf, 1996).
A deterioração do pescado se instala logo após a morte e avança com o tempo de exposição e estocagem do produto, sendo que a velocidade de decomposição depende de fatores exógenos (manipulação, manejo de abate e conservação) e endógenos (características físico-químicas do peixe) (Oetterer, 1998). Esses processos deteriorativos envolvem a atividade enzimática, a rancificação de gorduras e a ação de microorganismos presentes em sua superfície, guelras e trato intestinal (Leitão, 1994).

No músculo, a rancificação da gordura é causada por compostos químicos ou espécies reativas ao oxigênio que causam quebra das ligações duplas nas frações fosfolipídicas das membranas celulares, que no caso dos peixes são mais suscetíveis porque possuem maior grau de insaturação (Ruff et al., 2004). Isto prejudica sua fluidez e altera sua função como barreira semipermeável devido à perda de ácidos graxos poliinsaturados essenciais (AGPI) e à formação de hidroperóxidos, aldeídos e outros produtos tóxicos secundários (Sasaki et al., 2001; Wagner e Elmadfa, 2001).

O termo espécies reativas do oxigênio (EROS) inclui radicais livres como: radical hidroxila $\left(\mathrm{OH}^{\bullet}\right)$, radical óxido nítrico $\left(\mathrm{N}^{\bullet} \mathrm{O}\right)$, radical superóxido $\left(\mathrm{O}_{2}{ }^{\bullet-}\right)$, radical peroxila (LOO`) e não radicais como: peróxido de hidrogênio $\left(\mathrm{H}_{2} \mathrm{O}_{2}\right)$, ácido hipocloroso $(\mathrm{HClO})$, oxigênio "singlet" $\left({ }^{1} \mathrm{O}_{2}\right)$ e ozônio $\left(\mathrm{O}_{3}\right)$ (Aruoma, 1993); sendo que existem espécies mais reativas como a hidroxila, intermediárias como os radicais peroxilas e alquoxilas, e aquelas que reagem com poucas moléculas como $\mathrm{O}_{2}{ }^{--}$e $\mathrm{H}_{2} \mathrm{O}_{2}$ (Halliwell et al., 1995).

Os radicais livres são espécies químicas ou fragmentos moleculares com um ou mais elétrons desemparelhados em seu último orbital que lhes rendem alta reatividade devido à tendência de permanecerem pareados (Bryant, 2000). São formados e degradados por todos os 
organismos aeróbicos, produzidos em concentração fisiológica pelo funcionamento celular normal ou em excessivas quantidades sob estresse oxidativo (Matés, 2000; Imai e Nakagawa, 2003).

Em baixas concentrações, os EROS são benéficos e indispensáveis aos processos metabólicos normais, bem como nas defesas antimicrobianas, contribuindo com a atividade bactericida fagocítica (Puangkaw et al., 2005). Em contraste, altas doses ou remoção inadequada de EROS resultam no estresse oxidativo que pode causar severos danos metabólicos (Halliwell e Gutteridge, 1989).

A autoxidação pode ser dividida em três fases: iniciação, propagação $e$ terminação. A reação inicial envolve a geração de um radical livre (L') a partir de um ácido graxo insaturado (LH). Essa fase é muito lenta e depende de um iniciador (I), representado pelo calor, metais traços e certas enzimas catalisadoras (KamalEdin e Appelqvist, 1996).

$$
\text { Iniciação: I + LH } \rightarrow \mathrm{L}^{\cdot}+\mathrm{IH}
$$

A propagação inicia-se pela ação de uma espécie reativa que retira um átomo de hidrogênio do carbono central de uma estrutura pentadieno encontrada nas cadeias de ácidos graxos que contenham mais de uma ligação dupla. Ao contrário de sua molécula original, o radical lipídico gerado reage rapidamente com o oxigênio da atmosfera, formando um radical peróxido que novamente retirará um hidrogênio de outra cadeia acil, resultando em um hidroperóxido e uma nova espécie reativa ao oxigênio (FAO, 2003b), conforme as reações abaixo.

$$
\begin{gathered}
\text { Propagação: } \mathrm{L}^{\prime}+\mathrm{O}_{2} \rightarrow \mathrm{LOO} \\
\text { LOO }+\mathrm{LH} \rightarrow \mathrm{LOOH}+\mathrm{L}^{\circ}
\end{gathered}
$$

Nos ácidos graxos insaturados, livres ou esterificados, a abstração do átomo de hidrogênio é mais fácil no grupo metileno adjacente a sua dupla ligação (Sant'Ana e Mancini-Filho, 1995). Essa propagação continua até que o radical peróxido seja removido por uma reação com outro radical, formando produtos inativos ou não radicais (Burton e Traber, 1990).

Terminação: $\mathrm{LOO}^{*}+\mathrm{LOO}^{*} \rightarrow$ produtos inativos.

Os íons metálicos são muito importantes no processo inicial da autoxidação lipídica, pois catalisam a formação de espécies reativas do oxigênio como radicais hidroxilas $\left(\mathrm{OH}^{\circ}\right)$, por exemplo. Este radical reage imediatamente com lipídios e outras moléculas que estão próximas a onde ele foi gerado (FAO, 2003b). Os hidroperóxidos formados são rapidamente quebrados, em oxidações secundárias, produzindo aldeídos, cetonas, álcoois e ácidos carboxílicos que dão às carnes odores característicos e, em alguns casos, coloração amarelada (McCall e Frei, 1999).

\section{Métodos de Análise da Oxidação Lipídica}

Muitos métodos químicos e físicos têm sido propostos para quantificar a formação dos compostos resultantes da oxidação lipídica em carnes, como a determinação dos valores de peróxido, dienos conjugados (produzidos nos primeiros estágios da autoxidação), substâncias reativas ao ácido tiobarbitúrico (SRATB), teste de Kreis, HPLC (cromatografia de alta pressão), entre outros (Jardine et al., 2002). Os produtos mais freqüentemente medidos são hidroperóxidos e dienos conjugados, para a oxidação primária, e substâncias voláteis (SRATB) para a secundária (Moure et al., 2001). Dentre eles existem vantagens e desvantagens, porém os mais simples e rápidos são baseados na quantificação de pigmentos medidos espectrofotometricamente (Grau et al., 2000).

Em 1944, Koln e Liversedge observaram que tecidos animais incubados aerobicamente com ácido 2-tiobarbitúrico (TBA) produziam coloração rosa. Bernheim et al. (1948) descobriram que a coloração era resultado da formação de um complexo entre os compostos oxidados de gordura com o TBA. Essas descobertas 
deram origem ao teste mais utilizado na determinação da oxidação lipídica, o teste de TBA (Melton, 1983; Igene et al., 1985), sendo que a substância reativa ao ácido tiobarbitúrico presente em maior quantidade é o malonaldeído (MDA) (Kamal-Edin e Appelqvist, 1996), um dialdeído derivado do hidroperóxido. Porém, existem outros produtos da oxidação dos lipídeos, tais como, aldeídos insaturados e vários precursores não voláteis não identificados que também reagem com o TBA (Fernández et al., 1996).

A reação do teste de TBA envolve uma molécula de MDA que reage com duas moléculas de TBA, eliminando mais duas moléculas de água (Nawar, 1996), formando um pigmento cristalino rosa com absorbância entre 532 a 535 nm (Hoyland e Taylor, 1991).

Existem diferentes formas de medir as SRATB: pela reação direta da solução de TBA com os alimentos, por extração da amostra em solução ácida e por destilação. O primeiro método demanda alto gasto de tempo e solventes e, por isso, não é muito utilizado. O segundo é o mais recomendado e eficiente pela rapidez e simplicidade (Oetterer et al., 2004). A destilação, apesar de muito usada, resulta em valores mais altos, pois o aquecimento da amostra durante seu procedimento pode induzir à oxidação (Fernández et al., 1996; Ulu, 2004), necessitando da adição de antioxidante à amostra durante a análise.

$O$ teste de TBA também possui correlação positiva entre seus valores e o escore de rancificação avaliado pela análise sensorial, sendo apropriado na determinação do estado de oxidação lipídica em alimentos (Hoyland e Taylor, 1991).

\section{Defesas Antioxidantes em Peixes}

A convivência aparentemente harmoniosa dos organismos aeróbicos com os efeitos deletérios do oxigênio deve- se, primordialmente, ao desenvolvimento de mecanismos de defesa antioxidantes altamente especializados (Marcon, 1997). Halliwell e Gutteridge (1989) definiram os antioxidantes como substâncias capazes de, em concentrações relativamente baixas, competirem com outros substratos oxidativos e assim evitar ou inibir a sua oxidação. Devido aos efeitos danosos dos EROS, todas as células mantêm sistemas de defesas antioxidantes através de três níveis de proteção: prevenção da formação dos EROS; diminuição dos EROS através dos varredores (scavengers) de radicais livres ou enzimas antioxidantes e; reparo dos componentes celulares danificados (Sampaio, 2003).

Os peixes, assim como os vertebrados superiores, possuem os dois sistemas de defesa endógena: enzimático e não enzimático (Guerriero et al., 2002). O sistema enzimático é composto por constituintes primários, como as enzimas superóxido dismutase, catalase e glutationa peroxidase, e por enzimas de ação secundária, como a glutationa redutase, a glicose-6-fosfato desidrogenase e a glutationa S-transferase (Marcon, 1997).

O sistema de defesa antioxidante nãoenzimático desempenha papel importante na proteção de macromoléculas contra possível dano oxidativo, principalmente no plasma que possui pouca defesa enzimática. De acordo com sua solubilidade, está dividido em dois grupos: lipossolúveis ( $\alpha$-tocoferol, ubiquinol-10, $\beta$ caroteno) e hidrossolúveis (glicose, piruvato, ácido úrico, ácido ascórbico, bilirrubina, GSH) (Chan, 1996).

Os antioxidantes exógenos ou provenientes da nutrição (vitaminas $\mathrm{C}, \mathrm{E}$ e os carotenóides) e seus cofatores (cobre, manganês, zinco, selênio, ferro e a riboflavina), também mantêm equilíbrio na produção e controle das espécies reativas (Papas, 1999; Hamre et al., 2004). A sua ingestão pode ser um fator pré-abate importante na preservação da qualidade 


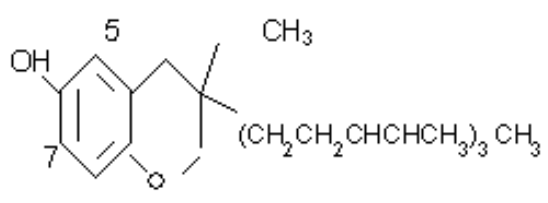

8

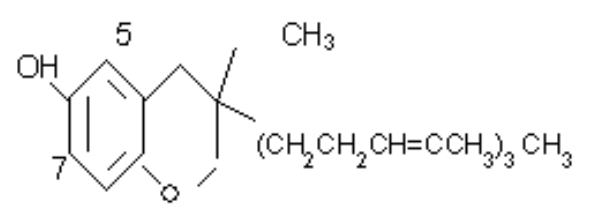

8

Estrutura básica dos tocóis

Estrutura básica dos trienóis

\begin{tabular}{ccc}
\hline $\mathrm{CH}_{3}$ & TOCOIS & TRIENOIS \\
\hline $5,7,8$ & $\alpha$ - tocoferol & $\alpha$ - tocotrienol \\
5,8 & $\beta$ - tocoferol & $\beta$ - tocotrienol \\
7,8 & $\gamma$-tocoferol & $\gamma$-tocotrienol \\
8 & $\delta$ - tocoferol & $\delta$ - tocotrienol \\
\hline
\end{tabular}

Figura 1 - Fórmula química da série tocoferol (Sant'Ana, 1998).

dos filés (Scaife et al., 2000), pois através da modificação da alimentação dos peixes, a qualidade do produto final pode ser mantida, evitando-se alterações na textura, cor, aroma e valor nutritivo decorrentes das reações oxidativas (Ruff et al., 2002).

$A$ vitamina $E$ é uma descrição genérica muito utilizada para lipídeos intimamente relacionados, os tocoferóis e tocotrienóis. $\mathrm{Na}$ natureza são encontradas oito formas de vitamina $E: \alpha, \beta, \delta, \gamma$ tocoferóis e tocotrienóis (Figura 1), que diferem entre si na localização do grupo metil do anel aromático de sua cadeia molecular (Devlin, 1997). Descoberta em 1922, e descrita como fator nutricional considerado especialmente importante na reprodução animal, apresenta como sua substância mais ativa, o tocoferol (Quinn e Wang, 1999).

Aparentemente, o $\alpha$-tocoferol é o tocoferol predominante em biomembranas animais e é mais efetivo na doação de elétrons devido à posição orto do seu grupo metil, quando comparado aos seus isômeros (Parker, 1989). Está presente em plantas e sementes, sendo encontrado em concentrações significantes nos óleos vegetais, gérmen ou farelo de glúten (Andriguetto, 1990).
A vitamina $E$ constitui a primeira linha de defesa dos sistemas biológicos. Atua principalmente protegendo as membranas dos compostos oxidáveis do citoplasma celular, fazendo a estabilização dos ácidos graxos insaturados e quebrando as cadeias de peróxidos (LOO) (Yamamoto et al., 2001). Previne a formação de hidroperóxidos lipídicos em seu estágio inicial através da doação de um átomo de hidrogênio a essa espécie reativa (Fang et al., 2002), resultando em um radical alfa-tocoferoxil (TO'). Esse radical será reduzido novamente à alfa-tocoferol (TOH) com a doação de elétrons pelo ácido ascórbico (Azzi e Stocker, 2000). Seguem abaixo, as equações que representam o mecanismo de ação dos tocoferóis.

$$
\begin{aligned}
& \mathrm{LOO}^{*}+\mathrm{TOH} \rightarrow \mathrm{LOOH}+\mathrm{TO}^{*} \\
& \mathrm{TO}^{*}+\mathrm{LOO}^{\cdot} \rightarrow \text { não radicais }
\end{aligned}
$$

A suplementação na dieta de $\alpha$ tocoferol tem sido a forma mais utilizada de vitamina $E$, pois sua localização específica na membrana torna-a muito eficiente quando comparada a outros antioxidantes (Botsoglou et al., 2003). Uma das hipóteses para sua maior eficiência seria a localização do seu anel cromanol na superfície polar dos 
fosfolipídios, com o lado da sua cadeia fitol interagindo com o acil da cadeia das gorduras insaturadas dos mesmos fosfolipídios, pela ação das forças de atração e dispersão de London Van der Wall's. Essa ligação protege os ácidos graxos poliinsaturados altamente oxidáveis da peroxidação por radicais produzidos pela enzima ligada à membrana adjacente, NADPH oxidase (Buckley e Morrissey, 1992).

\section{Efeito pró-oxidante da vitamina $E$}

As exigências vitamínicas dos peixes não são conhecidas com precisão e os níveis recomendados são médios. Quando a ingestão é inferior ao recomendado, pode induzir a um estado subnutrido. Níveis superiores ao exigido também apresentam o risco de aparecimento de estado tóxico (Sampaio, 2003).

Estudos sugerem que $0 \propto$-tocoferol, em particular, pode agir como pró-oxidante quando presente em altas concentrações nas dietas. Tokuda e Takeuchi (1995) têm sugerido que doses excessivas de $\propto-$ tocoferol nas dietas podem induzir a peroxidação lipídica em trutas. Kaewsrithong et al. (2001) observaram acúmulo de hidroperóxidos no sangue de peixes alimentados com $1000 \mathrm{mg}$ de vitamina $\mathrm{E} / \mathrm{kg}$ de dieta.

Entretanto, Kamal-Eldin e Appelqvist (1996) verificaram que os tocoferóis por si só não são pró-oxidantes, mas podem agir em sinergismo quando presentes em altas concentrações juntamente com outros próoxidantes, como metais de transição e peróxidos.

O efeito pró-oxidante do $\propto$-tocoferol está relacionado com a formação de radicais $\propto$-tocoferoxila ( $\propto$-TO) (Pokorny, 1987) que, quando presentes em altas concentrações, proporcionam a ocorrência de reações paralelas indesejáveis, podendo aumentar a taxa de peroxidação. $\mathrm{O} \propto-\mathrm{TO}$ reage com lipídios $(\mathrm{LH})$ e hidroperóxidos $(\mathrm{LOOH})$ pelo mecanismo de transferência de elétrons, gerando radicais alquilas e peroxilas, que reagirão com o oxigênio gerando outros radicais peróxidos, propagando a reação:

$$
\begin{gathered}
\propto-\mathrm{TO}^{\cdot}+\mathrm{LH} \rightarrow \propto-\mathrm{TOH}+\mathrm{L}^{\prime} \\
\propto-\mathrm{TO}^{\cdot}+\mathrm{LOOH} \rightarrow \propto-\mathrm{TOH}+\mathrm{LOO} .
\end{gathered}
$$

Esse efeito está relacionado com a baixa concentração de co-antioxidantes como a vitamina $\mathrm{C}$ nos tecidos dos peixes, que realizam a redução do radical alfa-tocoferoxil à alfa-tocoferol, com a doação de elétrons pelo ácido ascórbico. Altos níveis de vitamina $E$ nas dietas podem promover a peroxidação, aumentando a concentração de hidroperóxidos no sangue, sendo que a decomposição dos hidroperóxidos via unimolecular ou bimolecular parece ser responsável pela propagação de reações de autoxidação (Kaewsrithong et al., 2001):

$$
\begin{gathered}
\mathrm{LOOH} \rightarrow \mathrm{LO}^{\cdot}+\mathrm{OH}^{\cdot} \\
2 \mathrm{LOOH} \rightarrow \mathrm{LO}^{\cdot}+\mathrm{LO}_{2}+\mathrm{H}_{2} \mathrm{O}
\end{gathered}
$$

Esses radicais não desenvolvem significante papel prooxidantes no estágio inicial da oxidação, porque geralmente são neutralizados pelo $\propto$-tocoferol. No entanto, este mecanismo pode ser mais significativo quando a relação tocoferol: hidroperóxidos é igual ou menor a um (Kamal-Edin e Appelqvist, 1996). Porém, esses efeitos devem ser mais bem analisados, pois estudos que comprovem a ação pró-oxidante da vitamina $E$ ainda são escassos.

\section{Vitamina E para peixes}

Mesmo sendo reconhecida como nutriente essencial a todas as espécies animais, existem opiniões diferentes sobre seu nível ideal de suplementação nas dietas de peixes, pois esses são muito difíceis de serem determinados devido às inter-relações dessa vitamina com outros fatores, como: a quantidade de ácidos graxos poliinsaturados (Lopez- 
Bote, 2003), as características dos nutrientes e a presença de outras substâncias antioxidantes nos alimentos (Guo et al., 2001).

Segundo o National Research Council (NRC, 1993), o mínimo de vitamina E exigido para os peixes em geral é de 30 $\mathrm{mg} / \mathrm{kg}$ da dieta, e para tilápias é maior, de $50-100 \mathrm{mg} / \mathrm{kg}$ de ração. Porém, Shiau e Shiau (2001) observaram que a necessidade de vitamina $E$ para tilápias dependia da quantidade de lipídio nas rações utilizadas, concluindo que tilápias alimentadas com dietas contendo $5 \%$ e $12 \%$ de lipídios necessitam de 40-44 e 60$66 \mathrm{mg}$ de vitamina $\mathrm{E} / \mathrm{kg}$ de dieta, respectivamente.

Apesar da estreita relação entre vitamina $E$ e a gordura da alimentação, trabalhos anteriores já pesquisavam a utilização desse antioxidante nas dietas de peixes, independente de sua inter-relação com outros nutrientes. Em 1990, Frigg et al. observaram que trutas alimentadas com níveis de 100 a $200 \mathrm{mg}$ de vitamina $\mathrm{E} / \mathrm{kg}$ de ração) apresentaram melhor desempenho do que o grupo controle, apresentando níveis elevados de deposição de tocoferol nos tecidos. Gatlin III et al. (1992) encontraram resultados similares a esses estudos, observando que a suplementação de vitamina $\mathrm{E}$ em níveis acima de $240 \mathrm{mg} / \mathrm{kg}$ nas dietas aumentou a concentração de tocoferol nos tecidos, e proporcionou menor oxidação lipídica dos filés, após processamento.

O aumento da quantidade de vitamina E no músculo e sua interação com o sistema antioxidante dos peixes foram analisados por Tocher et al. (2002), que observaram que menores quantidades de vitamina $E$ nas dietas levavam a diminuição de seus níveis nos músculos e ao aumento da atividade oxidante do organismo, produzindo altos níveis de peróxidos lipídicos.

A partir dessas observações, ficou claro que o papel antioxidante da vitamina $E$ nas dietas de organismos aquáticos também era o de garantir a qualidade do pescado, melhorando a conservação, e conseqüentemente, a textura dos filés e dos produtos derivados de peixes (Bad II e Howell, 2002).

Dessa forma, diversos trabalhos foram realizados com a suplementação de diferentes níveis de vitamina $\mathrm{E}$ em dietas para salmões do atlântico (Onibi et al., 1996; Hamre et al., 2004), robalos (Pirini et al., 2000; Gatta et al., 2000), pacus (Sant'Ana, 1998; Sant'Ana e Fernandes, 2000) e pregados (Ruff et al., 2002b; 2003), sendo que todos descreveram redução nas taxas de oxidação e melhor conservação desses peixes durante a estocagem.

Atualmente, os estudos têm focado diferentes formas de utilização da vitamina E, comparando sua ação antioxidante quando adicionada às dietas ou simplesmente incorporada aos produtos após o abate e processamento. Fogaça e Sant'Ana (2007) compararam os efeitos da utilização de $\alpha$-tocoferil nas dietas ou diretamente no músculo post mortem, e sua interação, ou seja, adição in vivo juntamente com a post mortem, e observaram que a eficiência é melhor na utilização in vivo, em níveis de suplementação entre 100 a $200 \mathrm{mg}$ de tocoferol/kg de ração para tilápias.

O desafio agora está em determinar qual a melhor forma de utilização, e qual forma de vitamina $E$, o acetato de $\alpha$ tocoferil ou de a-tocoferol, seria mais adequada para uso in vivo e post mortem. Além do tempo de administração na dieta, pois a maioria dos trabalhos experimentou suplementação por períodos de aproximadamente 65 dias, na fase de terminação dos cultivos.

\section{CONSIDERAÇÕES FINAIS}

A taxa de oxidação lipídica depende de inúmeros fatores, incluindo a composição de ácidos graxos poliinsaturados e a concentração de 
agentes pró e antioxidantes que podem ser manipulados pela dieta. Sua prevenção pode ser feita através da utilização de vitamina $E$ nas dietas de organismos aquáticos, sendo comprovadamente eficiente na conservação do pescado durante o processamento e estocagem.

Novos estudos devem ser conduzidos no sentido de preencher as lacunas sobre as formas e o tempo de utilização da vitamina $E$ em dietas para organismos aquáticos, levando-se em consideração seus benefícios antioxidantes no desempenho, na imunidade e na qualidade final dos peixes beneficiados.

\section{REFERÊNCIAS}

ANDRIGUETTO, J.M. Nutrição animal. 4. ed. São Paulo: Nobel, 1990. 395p.

ARUOMA, O.I. Free radicals and food. Chemistry in Britain, v.29, n.3, p.210-214, 1993.

AZZI, A.; STOCKER, A. Vitamin E: non-antioxidant roles. Progress in Lipid Research, v.39, p.231255, 2000.

BAD II, F.; HOWELL, N.K. Effect of antioxidants, citrate and cryoprotectants on protein denaturation and texture of frozen cod (Gadus morhua). Journal of Agriculture and Food Chemistry, v.50, n.7, p.2053-2061, 2002.

BERNHEIM, F.M.; BERNHEIM, L.C.; WILBUR, K.M. The reaction between thiobarbituric acid on the oxidation products of certain lipids. Journal of Biology and Chemistry, v.174, p.257-264, 1948.

BORGHETTI, J.R. Aqüicultura: uma visão sobre a produção de organismos aquáticos no Brasil e no Mundo. Curitiba: GIA, 2003. 129p.

BOTSOGLOU N.A. et al. Inhibition of lipid oxidation in long-term frozen stored chicken meat by dietary oregano essential oil and a-tocopheryl acetate supplementation. Food Research International, v.36, p.207-213, 2003.

BRYANT R.J. Evidence that diet modification reduces oxidation damage in cells. Food Rap, v.10, p.10, 2000.

BUCKLEY, D.J.; MORRISSEY, P.A. Vitamin E and meat quality. In: Hoffmann, F. Vitamins and fine chemicals. Nova York: La Roche, 1992. Não paginado.

BURTON, G.W.; TRABER, M.G. Vitamin E: antioxidant activity, biokinetics and bioavaibility.
Annual Review of Nutrition, v.10, p.357-382, 1990.

CHAN, S.S. Estudo morfofuncional de leucócitos polimorfonucleares sanguíneos como parâmetro de estresse oxidativo. 1996, São Paulo, 230p. Dissertação (Mestrado em Parasitologia Animal) - Faculdade de Medicina Veterinária e zootecnia da Universidade de São Paulo.

DEVLIN, T.M. Manual de bioquímica com correlações clínicas. 4 ed. São Paulo: Edgard Blucher, 1997. 1007p.

FANG, Y.Z.; YANG, S.; WU, G. Free Radicals, Antioxidants, and Nutrition. Nutrition, v.18, p.872879, 2002.

FAO. Quality and quality changes in fresh fish: post mortem changes in fish. Roma: $F A O$, 2003b. Disponível em <http://www.fao.org/docrep/v7180e/v7180e06.htm > Acesso em: 01/nov/2003.

FAO. Revisión del Estado Mundial de la Acuicultura. Roma: FAO, 2003a, $103 \mathrm{p}$. Disponível em <http://www.fao.org> Acesso em: 01/jun/2005.

FAOSTAT. 2006. FAO Statistical Yearbook 2007- 2008. Disponível em <http://www.faostat.fao.org> Acesso em: 31/ago/2009.

FERNÁNDEZ, J.; PÉREZ-ALVAREZ, J.A.; FERNÁNDEZ-LÓPEZ, J.A. Thiobarbituric acid test for monitoring lipid oxidation in meat. Food Chemistry, v.59, n.3, p.345-353, 1996.

FOGAÇA, F.H.S.; SANT'ANA, L.S. Tocopherol in the lipid stability of tilapia (Oreochromis niloticus) hamburgers. Food Chemistry, v.105, p.1214 1218, 2007.

FRIGG, M.; PRABUCKI, A.L.; RUHDEL, E.U. Effect of dietary vitamin $E$ levels on oxidative stability of trout fillets. Aquaculture, v.84, p.145158, 1990.

GATLIN III, D.M.; BAI, S.C.; ERICKSON, M.C. Effects of dietary vitamin $E$ and synthetic antioxidants on composition and storage quality of channel catfish, Ictalurus punctatus. Aquaculture, v.106, p.323 - 332, 1992.

GATTA, S.S. et al. The influence of different levels of dietary vitamin e on sea bass (Dicentrarchus labrax) flesh quality. Aquaculture Nutrition, v.6, n.1, p.47, 2000.

GRAU, A. et al. Measurement of 2-thiobarbituric acid values in dark chicken meat through derivated spectrophotometry: influence of various 
parameters. Journal of Agriculture and Food Chemistry, v.48, p.1155-1159, 2000.

GUERRIERO, G.; DI FINIZIO, A.; GARCIA, G. Stress induced changes of plasma antioxidants in aquatic sea bass. Compendium of Biochemistry and Physiology, part. A, v.132, p.205-211, 2002.

GUO, Y. et al. Effects of supplementation with vitamin $E$ on the performance and the tissue peroxidation of broiler chicks and the stability of thigh meat against oxidative deterioration. Animal Feed Science and Technology, v.89, n.3-4, p.165173, 2001.

HALLIWELL, B. et al. Free radicals and antioxidants in food in vivo: what they do and how they work. Reviews of Food Science and Nutrition, v.35, n.1/2, p.7-20, 1995.

HALLIWELL, B.; GUTTERIDGE, J.M.C. Free radicals in biology and medicine. 2 ed. Oxford: Claredon, 1989. 469p.

HAMRE, K. et al. Antioxidant vitamins, minerals and lipid levels in diets for Atlantic Salmon (Salmo salar, L.): effects on growth performance and fillet quality. Aquaculture Nutrition, v.10, p.113-123, 2004.

HOYLAND, D. V.; TAYLOR, A. J. A Review of the methodology of the 2-thiobarbituric acid test. Food Chemistry, v.40, p.271-291, 1991.

IGENE, J. O. et al. Evaluation of 2-thiobarbituric acid reactive substances in relation to warmed over flavour development in cooked chicken. Journal of Agriculture and Food Chemistry, v.33, p.364-367, 1985.

IMAI, H.; NAKAGAWA, Y. Biological significance of phospholipid hydroperoxide glutathione peroxidase (PHG Px, GPx4) in mammalian cells. Free

Radicals and Biology Medicine, v.34, n.2, p.145169, 2003.

JARDINE, D. et al. Liquid chromatography-mass spectrometry (LC-MS) investigation of the thiobarbituric acid reactive substances (TBARS) reaction. Journal of Agriculture and Food Chemistry, v.50, p.1720-1724, 2002.

JENSEN, C.; LAURIDSEN, C.; BERTELSEN, G. Dietary Vitamin E: Quality and Storage Stability of Pork and Poultry. Trends in Food Science and technology, v.9, n.2, p.62-72, 1998.

KAEWSRITHONG, J. et al. Effects of an excess dose of dietary alpha-tocopherol on hydroperoxide accumulation and erythrocyte osmotic fragility of sweet plecoglossus altivelis (Temminck et Schleget). Aquaculture Research, v.32, p.191198, 2001.
KAMAL-ELDIN, A.; APPELQVIST, L.A. The chemistry and antioxidant properties of tocopherols and tocotrienols. Lipids, v.31, n.7, p.671-701, 1996.

KOLN, H.I.; LIVERSEDGE, M. On a new aerobic metabolite whose production by brain is inhibited by apomorphene, emetine, epinephrine and menadione. Journal of Pharmacology, v.82, p.292-297, 1944.

LEITÃO, M. F. de F. Microbiologia e deterioração do pescado fresco e refrigerado de origem fluvial ou marinha. In: Simpósio sobre controle de qualidade microbiológico, químico, físico e organoléptico de pescado e derivado, 1994, Campinas. Anais... Campinas: ITAL, 1994. p.1126.

LÓPEZ -BOTE, O.J. Effect of vitamin e supplementation and partial substitution of poly with mono-unsaturated fatty acids in pigs diets on muscle, and microssome extract alpha-tocopherol concentration and lipid oxidation. Animal Science, v.57, n.1, p.11-25, 2003.

MACEDO-VIEGAS, E.M.; SOUZA, M.L.R. Préprocessamento e conservação do pescado produzido em piscicultura. In: CYRINO, J.E.P.; URBINATI, E.C.; FRACALOSSI, D.M.;

CASTAGNOLLI, N. Tópicos Especiais em Piscicultura de Água Doce Tropical Intensiva. 1 ed. São Paulo: Funep, 2004. Cap. 14, p.405-480.

MARCON, J. L. Estresse oxidativo em duas espécies de teleósteos amazônicos, Astronotus ocellatus e Colossoma macropomum, expostos a diferentes tensões de oxigênio: uma abordagem comparativa. 1997, Manaus, 103 p. Tese (Doutorado em Biologia Tropical e Recursos Naturais do INPA, área de concentração em Biologia de Água Doce e Pesca Interior) - Instituto Nacional de Pesquisas da Amazônia, INPA, Universidade Federal do Amazonas, UFAM.

MATÉS, J.M. Effects of antioxidant enzymes in the molecular control of reactive oxygen species toxicology. Toxicology, v.153, p.83-104, 2000.

McCALL M.R.; FREI B. Can antioxidant vitamins materially reduce oxidative damage in humans? Free Radicals and Biology Medicine, v.26, n.7/8, p.1034-1053, 1999.

MELO FRANCO, B. D. G.; LANDGRAF, M. Microbiologia dos alimentos. São Paulo: Atheneu, 1996. 182p.

MELTON, S. L. Methodology for following lipid oxidation in muscle foods. Food Technology, v.37, p.105-116, 1983. 
MORRISSEY, P. A. et al. Lipid in meat and meat product. Meat Science, v.49, sup.1, p.73-86, 1998.

MOURE, A. et al. Natural antioxidants from residual sources. Food Chemistry, v.72, p.145-171, 2001.

NATIONAL RESEARCH COUNCIL (NRC). Nutrient requirements of fish. Washington: Academic Press, 1993. 114p.

NAWAR, W.W. Lipids. In: FENNEMA, O.R. Food Chemistry. 3 ed. New York: Marcel Dekker, 1996. Cap.5, p.226-319.

NOGALA-KALUCKA, M. et al. Changes in antioxidant activity and free radical scavenging potential of rosemary extract and tocopherols in isolated rapeseed oil triacylglycerols during accelerated tests. Food Chemistry, v.93, p.227235, 2005.

OETTERER, M.; SIQUEIRA, A.A.Z.C.; GRŸSCHEK, S.B. Tecnologias emergentes para processamento do pescado produzido em piscicultura. In: CYRINO, J.E P.; URBINATI, E.C.; FRACALOSSI, D.M.; CASTAGNOLLI, N. Tópicos especiais em piscicultura de água doce tropical intensiva. 1 ed. São Paulo: Funep, 2004. Cap. 15, p. 481-500.

OETTERER, M. Técnicas de beneficiamento e conservação do pescado de água doce. Panorama da Aqüicultura, v.8, n.46, p.14-20, 1998.

ONIBI G.E.; SCAIFE J.R.; FLETCHER T.C. Influence of a-tocopherol acetate in high lipid diets on quality of refrigerated Atlantic Salmon (Salmo salar) fillets. Refrigeration and Aquaculture, v.33, p.145-152, 1996.

PAPAS, A.M. Diet and Antioxidant Status. Food Chemistry and Toxicology, v.37, p.999-1007, 1999.

PARKER, R.S. Dietary and biochemical aspects of vitamin E. Advocate Food Nutrition and Research, v.33, p.157-232, 1989.

PIRINI M. et al. Effect of refrigerated storage on muscle lipid quality of sea bass (Dicentrarchus labrax) fed on diets containing different levels of vitamin E. Food Chemistry, v.68, p.289-293, 2000.

POKORNY, J. Major factors affecting the autoxidation of lipids. In: CHAN, H. W. S. Autoxidation of Unsaturated Lipids. London: Academic Press, 1987. p.141-206.

PUANGKAW, J. et al. T. Antioxidant defense of rainbow trout (Oncorhynchus mykiss) in relation to dietary n-3 highly unsaturated fatty acids and vitamin $\mathrm{E}$ contents. Compendium of Biochemistry and Physiology, v.140, p.187-196, 2005.
QUINN, P.J.; WANG, X. Vitamin E and its function in membranes. Progress in Lipid Research, v.38, p.309-336, 1999.

RUFF, N. et al. The effect of dietary vitamin $E$ and $C$ level on market-size turbot (Scophthalmus maximus) fillet quality. Aquaculture Nutrition, v.9, n.2, p.91, 2003.

RUFF, N. et al. Slaughtering method and dietary tocopherol acetate supplementation affect rigor mortis and fillet shelf-life of turbot (Scophthalmus maximus). Aquaculture Research, v.33, n.9, p.703, 2002.

RUFF, N. et al. Distribution of $\alpha$-tocopherol in fillets of turbot (Scophthalmus maximus) and Atlantic halibut (Hippoglossus hippoglossus), following dietary a-tocopheryl acetate supplementation. Aquaculture Nutrition, v.10, p.75-81, 2004.

SAMPAIO, $F$ G. Selênio e vitamina $E$ em dietas para a tilápia do Nilo Oreochromis niloticus. 2003, Botucatu, 99 p. Dissertação (Mestrado em Nutrição e Produção Animal) - Faculdade de Medicina Veterinária e Zootecnia, FMVZ, UNESP.

SANT'ANA, L.S.; MANCINI-FILHO, J. Influence of the addition of antioxidants in vivo on the fatty acid composition of fish fillets. Food Chemistry, v.68, p.175-178, 2000.

SANT'ANA, L.S.; FERNANDES, J.B. Efeito do armazenamento na composição em ácidos graxos de filés de peixes da espécie pacu (Piaractus mesopotamicus). In: Congresso Brasileiro de Ciência e Tecnologia de Alimentos, 2000 , Fortaleza. Anais... Fortaleza: SBCTA, 2000. v.4, p.5-272.

SANT'ANA, L.S. Influência da Fonte Lipídica da dieta e da Adição de Antioxidantes in vivo na Composição em ácidos graxos de filés de Pacu (Piaractus mesopotamicus) cultivados. 1998, São Paulo, 157 p. Tese (Doutorado em Ciências Farmacêuticas) - Faculdade de Ciências Farmacêuticas da Universidade de São Paulo, USP.

SANT'ANA, L.S.; MANCINI-FILHO, J. Mecanismos de Proteção Oxidativa na Utilização de Antioxidantes in vivo em Músculos Animais.

Cadernos de Nutrição, n.10, p.48-63, 1995.

SASAKI, K.; MITSUMOTO, M.; KAWABATA, K. Relationship between lipid peroxidation and fat content in Japanese Black beef Longissimus muscle during storage. Meat Science, v.59, n. 4, p.407-410, 2001.

SCAIFE, J.R. et al. Influence of a-tocopherol acetate on the short- and long-term storage properties of fillets from Atlantic salmon Salmo 
salar fed a high lipid diet. Aquaculture Nutrition, v.6, p.65-71, 2000.

SHIAU, S.Y.; SHIAU, L.F. Re-evaluation of the vitamin $\mathrm{E}$ requirement of juvenile Tilapia (Oreochromis niloticus $x$ O. aureus). Animal Science, v.72, p.529-534, 2001.

TOCHER D.R. et al. Effects of dietary vitamin $E$ on antioxidant defense mechanism of juvenile turbot (Scophthalmus maximus L.) and sea bream (Sparus aurata L.) Aquaculture Nutrition, v.8, n.3, p.195, 2002.

TOKUDA, M.; TAKEUCHI, M. Effects of excess doses alphatocopherol on the lipids and function of rainbow trout liver. Journal of Nutrition and Science Vitaminology, v.41, p.25-32, 1995.
ULU, H. Evaluation of three 2-thiobarbituric acid methods for the measurement of lipid oxidation in various meats and meats products. Meat Science, v.67, p.683-687, 2004.

WAGNER, K-H.; ELMADFA, I. Effects of tocopherols and their mixtures on the oxidative stability of olive oil and linseed oil under heating. Journal of Lipid Science and Technology, v.103, p.624-629, 2001.

YAMAMOTO, Y. et al. An unusual vitamin E constituent provides enhanced antioxidant protection in marine organisms adapted to coldwater environments. Procedures of National Academic of Sciences, v.98, p.13144-13148, 2001. 